\title{
Shaking conditions required for flame structure formation in a water-immersed granular medium
}

\author{
Nao Yasuda and Ikuro Sumita*
}

\begin{abstract}
Flame structures found in sedimentary rocks may have formed from liquefaction and gravitational instability when the sediments were still unconsolidated and were subject to shaking caused by earthquakes. However, the details of the process that leads to the formation of the flame structure, and the conditions required for the instability to initiate and grow remain unclear. Here, we conduct a series of small-scale laboratory experiments by vertically shaking a case containing a water-immersed layered granular medium. The upper granular layer consists of finer particles and forms a permeability barrier against the interstitial water which percolates upwards. We shake the case sinusoidally at different combinations of acceleration and frequency. We find that there is a critical acceleration above which the instability develops at the two-layer interface. This is because the upward percolating water temporarily accumulates beneath the permeability barrier. For larger acceleration, the instability grows faster and the plumes grow to form a flame structure, which however do not completely penetrate through the upper layer. We classify the experimental results according to the final amplitude of the instability and construct a regime diagram in the parameter space of acceleration and frequency. We find that above a critical acceleration, the instability grows and its amplitude increases. Moreover, we find that the critical acceleration is frequency dependent and is smallest at approximately $100 \mathrm{~Hz}$. The frequency dependence of the critical acceleration can be interpreted from the combined conditions of energy and jerk (i.e., the time derivative of acceleration) of shaking, exceeding their respective critical values. These results suggest that flame structures observed in sedimentary rocks may be used to constrain the shaking conditions of past earthquakes.
\end{abstract}

Keywords: Liquefaction; Earthquake; Flame structure; Permeability barrier; Laboratory experiment

\section{Background}

Liquefaction and related phenomena, such as the outflow of ground water, sand boils, mud volcanism, and ground subsidence, are commonly observed after earthquakes (Manga and Wang 2007). These phenomena reflect the consequences of loosening of particle contacts, pore pressure increase, upward percolation of pore water, and subsequent compaction of particles. When a layer consisting of fine particles (e.g., clay, mud) exists above a coarse particle (e.g., sand) layer, it forms a permeability barrier. This can help increase pore pressure and result in liquefaction because the expelled water temporarily accumulates at the barrier (Allen 1985). These phenomena

*Correspondence: isumita@staff.kanazawa-u.ac.jp

Earth and Environmental Sciences Course, Division of Natural System, Graduate School of Natural Science and Technology, Kanazawa University, Kakuma, Kanazawa 920-1192, Japan can occur whenever liquid is present; indeed, topographic features which suggest mud volcanism have even been discovered on Mars (Oehler and Allen 2010; Pondrelli et al. 2011). Evidence of past earthquake-induced liquefaction may also be preserved in sedimentary rocks, and the resulting structures are commonly known as seismites (e.g., Montenat et al. 2007). One possible example is the flame structure (see Figure 1), which may have formed from liquefaction and subsequent gravitational instability when the sediments were still at the ocean floor.

There is considerable evidence which indicate that there is a critical condition for liquefaction and subsequent instability to occur. Compilation of liquefaction-related phenomena shows that there is a minimum seismic magnitude, which increases with epicentral distance, required for these phenomena to occur (Manga and Brodsky 2006;

\section{Springer}

(C) 2014 Yasuda and Sumita; licensee Springer. This is an Open Access article distributed under the terms of the Creative

Commons Attribution License (http://creativecommons.org/licenses/by/2.0), which permits unrestricted use, distribution, and reproduction in any medium, provided the original work is properly credited. 


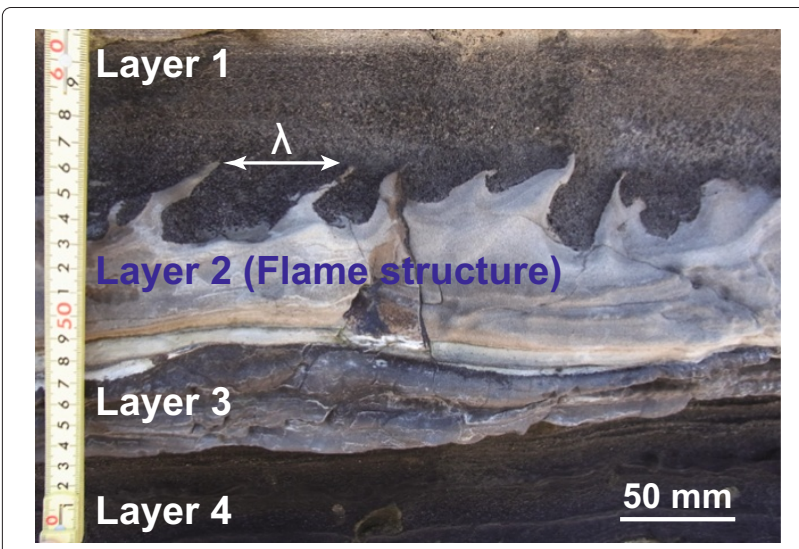

Figure 1 Flame structure in a sedimentary rock. Flame structure in a tuff layer (layer 2) observed in an outcrop on Jogashima Island, Kanagawa, Japan. The thickness of the layer 2 is approximately $60 \mathrm{~mm}$, and the wavelength of the flame structure is $\lambda \sim 40 \mathrm{~mm}$. The measured densities of the particles comprising these layers are as follows: layer $1=2,340$; layer $2=2,140$; layer $3=2,050$; layer $4=2,230\left(\mathrm{~kg} / \mathrm{m}^{3}\right)$.

Manga and Wang 2007). The threshold at which liquefaction occurs has also been investigated using laboratory experiments. For example, oscillatory deformation experiments of water-saturated granular matter and simultaneous pore-water pressure measurements have been conducted to constrain the strain needed for liquefaction (e.g., Vucetic 1994). Experiments for the situation in which a permeability barrier exists have also been conducted to study the formation process of water film and sand boils. In these studies, a one-dimensional tube test, to which an instant shock is applied (Kokusho 1999; Kokusho and Kojima 2002), or horizontal shake tables (Kokusho 1999; Yamaguchi et al. 2008) were used. Experiments more closely simulating natural conditions have also shown that a variety of structures can form as a consequence of shaking (Moretti et al. 1999).

On the other hand, the response of a dry granular matter under vertical shaking has intrigued physicists for a long time. Experiments have revealed instabilities such as surface waves, the Brazil nut effect, and granular convection (Duran 2000). Detailed parameter studies have shown that a variety of instabilities can occur, depending on the combination of acceleration and frequency of shaking (Burtally et al. 2002). Recently, shaking conditions required for granular convection have been investigated (Hejmandy et al. 2012). Experiments for liquid-saturated cases have also been conducted and have revealed that a number of novel phenomena can occur (e.g., Schleier-Smith and Stone 2001; Voth et al. 2002; Leaper et al. 2005; Clement et al. 2009, 2010).

Here, we consider the situation observed in Figure 1 and attempt to explain how the observed structures may have formed, and consider constraining the required shaking conditions. In order to answer these questions, we conduct a series of experiments in which a water-immersed granular medium with a permeability barrier is shaken vertically under a range of accelerations and frequencies.

\section{Methods}

Figure 2 shows the experimental setup. We use a styrol case with a width of $99.4 \mathrm{~mm}$, a height of $H=107.6 \mathrm{~mm}$, and a thickness of $22.0 \mathrm{~mm}$, which is filled with a mixture of glass beads and water. We add $0.25 \mathrm{ml}$ of surfactant to the water so that the glass beads do not form clusters. The lowermost $33.8 \pm 0.5 \mathrm{~mm}$ of the cell consists of a glass beads layer comprised of two size-graded layers. The upper layer (thickness $9.8 \pm 0.3 \mathrm{~mm}$ ) consists of fine particles (white) with a diameter of $d=0.05 \pm 0.01 \mathrm{~mm}$, a density of $\rho=2,500 \mathrm{~kg} / \mathrm{m}^{3}$, and a volumetric particle packing fraction of $\phi=0.60 \pm 0.02$. The lower layer (thickness $24.0 \pm 0.4 \mathrm{~mm}$ ) consists of coarse particles (red) with $d=0.22 \pm 0.04 \mathrm{~mm}, \rho=2,500 \mathrm{~kg} / \mathrm{m}^{3}$, and $\phi=0.49 \pm 0.01$. The thicknesses of the upper and lower layers scaled by the particle diameters are $N_{\text {upper }} \sim 200$ and $N_{\text {lower }} \sim 110$, respectively. The values of $\phi$ for the upper and lower layers are smaller than $\phi \sim 0.64$ for dense random close packing of spheres (Mavko et al. 1998), indicating that the particles are loosely packed. After shaking for $5 \mathrm{~s}$, compaction occurs and the packing fraction of the glass bead layer as a whole increases from $\phi=0.52 \pm 0.01$ at $t=0 \mathrm{~s}$ to $\phi=0.54 \pm 0.02$ at $t=9.9 \mathrm{~s}$.

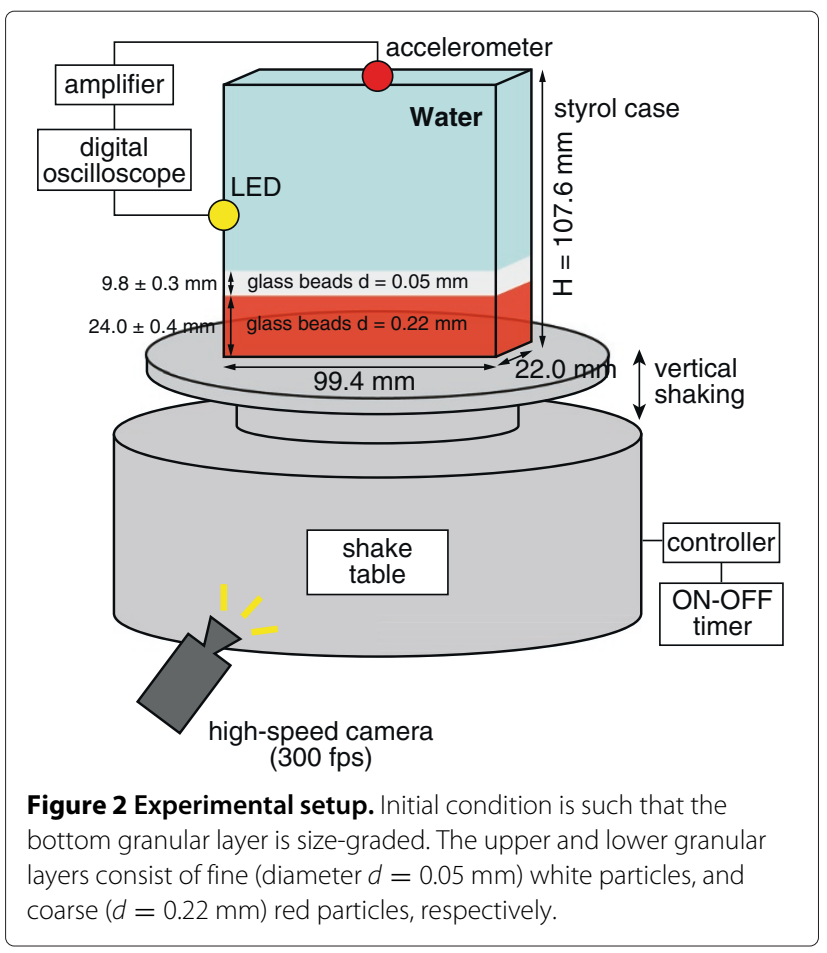


The above particle size ratio is chosen so that it is sufficiently large to result in an efficient size grading from settling, but not so large that the small $(d=0.05 \mathrm{~mm})$ particles do not fit into the pore space of the large $(d=0.22 \mathrm{~mm})$ particles. Particle settling velocity $V_{\mathrm{s}}$ can be estimated from the Stokes settling formula

$$
V_{\mathrm{s}}=\frac{\Delta \rho g d^{2}}{18 \eta}
$$

where $\Delta \rho$ is the particle-water density difference, $g$ is the gravitational acceleration, and $\eta$ is the viscosity of water. An efficient size grading occurs for a mixture of $0.05 \mathrm{~mm}$ and $0.22 \mathrm{~mm}$ particles, which can be understood as follows. The approximate fraction $F$ of the small particles that can be included in the lower layer (consisting primarily of large particles) can be estimated using the time scale for a particle to settle $\sim H / V_{\mathrm{s}}$. Assuming that the particles are initially homogeneously distributed, the fraction $F$ of the small particles that have settled during the time needed for all of the large particles to settle is $F \sim$ $\left(H / V_{\mathrm{s}(\mathrm{la})}\right) /\left(H / V_{\mathrm{s}(\mathrm{sm})}\right) \simeq 0.06$, where the subscripts sm and la indicate small and large particles, respectively. A small $F(\ll 1)$ value indicates an efficient size grading. We also note that, since permeability scales as $\propto d^{2}$ (e.g., Andreotti et al. 2013), this particle size ratio results in a permeability ratio of 19 , and the upper layer becomes a low permeability layer.

The experimental procedure is as follows. We first thoroughly shake the cell by hand and allow the particles to settle for $300 \mathrm{~s}$, which is 5.7 times longer than the settling time of the $0.05 \mathrm{~mm}$ particles $\left(H / V_{\mathrm{s}}=53 \mathrm{~s}\right)$. The cell is then attached to a shake table (Big Wave, Asahi Seisakusyo, Tokyo, Japan) and is shaken vertically for $5 \mathrm{~s}$. The vertical displacement $z$ changes with time $t$ as

$$
z=A \sin (2 \pi f t)
$$

where $A$ is the amplitude and $f$ is the frequency. An accelerometer (352A24, PCB Piezotronics, New York, USA) is attached to the case, and the signal is amplified (482C, PCB Piezotronics, New York, USA) and recorded by a digital oscilloscope (ZR-RX70, Omron, Kyoto, Japan). We use a high-speed camera (EX-F1, Casio, Tokyo, Japan) at $300 \mathrm{fps}$ to record the motion within the cell. An LED lamp is used to synchronize the acceleration and image data.

We calculate the acceleration from the voltage output of the accelerometer. Here, we calculate the $\sqrt{2} \times$ rootmean-square of the acceleration data to obtain the average peak acceleration. This gives the exact peak acceleration for a sinusoidal shaking.

We conduct experiments under a peak acceleration range of 1.4 to $78.3 \mathrm{~m} / \mathrm{s}^{2}$ and a frequency range of 10 to $5,000 \mathrm{~Hz}$. For comparison, this frequency range extends beyond the typical high frequency response limit (approximately $10 \mathrm{~Hz}$ ) of seismometers (Shearer 1999). Digital images are analyzed using MATLAB. The spatial resolution of the images is $0.118 \pm 0.002 \mathrm{~mm}$ per pixel. We binarized the images for the time interval of $t=0$ to $9.9 \mathrm{~s}$ to obtain the heights of the granular layer surface and the two-layer interface, and analyzed how these heights change with time.

\section{Results}

\section{Growth of an instability}

Figure 3 and Additional file 1 show a typical example of an experiment in which the instability develops at the two-layer interface and a flame structure forms. We start shaking the case at $t=0 \mathrm{~s}$ and find that the heights of the lower and upper layers decrease with time. Initially the two-layer interface remains flat. This indicates that the interstitial water is percolating upwards in a laterally uniform manner, and the granular medium is compacting. After $t \sim 0.5 \mathrm{~s}$, undulations start to develop. The photo at $t=1.6 \mathrm{~s}$ in Figure 3a shows the occurrence of sand boils at the surface of the granular layer, indicating an upward channelized flow. Figure $3 \mathrm{~b}$ is a close-up image at $t=1.6 \mathrm{~s}$, indicating the formation of an instability with a wavelength of $\lambda \sim 2.7 \mathrm{~mm}$. Since this instability forms at the permeability barrier, we interpret this as the onset of a Rayleigh-Taylor type instability, which occurred as a consequence of the formation of a thin buoyant liquefied layer at the two-layer interface. The amplitude of the instability increases with time (Figure 3d), and undulations merge to form plumes (Figure 3c). However, the amplitude growth is suppressed and the plumes do not fully penetrate through the upper layer, in contrast to the Rayleigh-Taylor type instability of superimposed viscous fluids (Whitehead and Luther 1975). Here, we note that the shape of the flame structure deviates from the sinusoidal shape and is characterized by upward pointing cusps.

Figure 4a shows how the heights of the granular layer surface and the two-layer interface evolve with time for the same experiment shown in Figure 3. Here, thin vertical sections of the images, which are separated by a time interval of $0.033 \mathrm{~s}$, are aligned horizontally as a function of time. The figure shows that in the initial stage, both the heights of the granular layer surface and the two-layer interface decrease with time as a result of compaction. The instability starts to grow at $t \sim 0.5 \mathrm{~s}$, but the height of the two-layer interface starts to increase at a later time, $t \sim 1.8 \mathrm{~s}$. This is because the instability growth and compaction occur simultaneously, and the height increase of the two-layer interface does not commence until the former exceeds the latter. Figure $4 \mathrm{~b}$ shows the growth of the interfacial amplitude $(\delta z)$ with time which was calculated as follows. We define the height at $t=0$ as the 
a

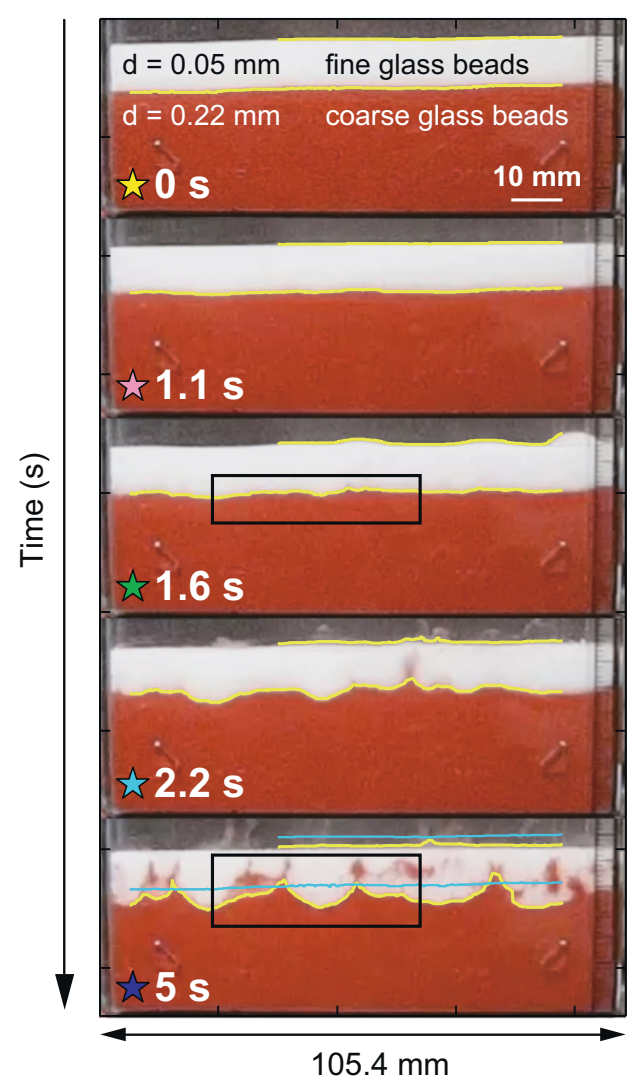

b

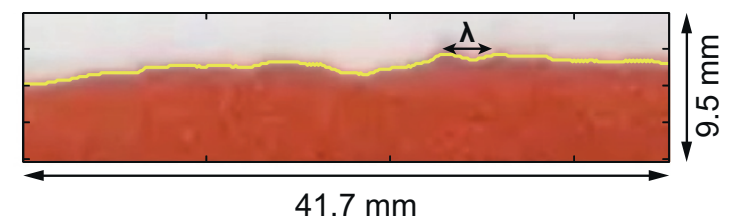

C

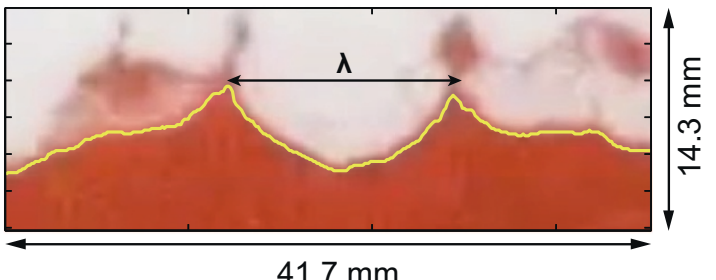

d

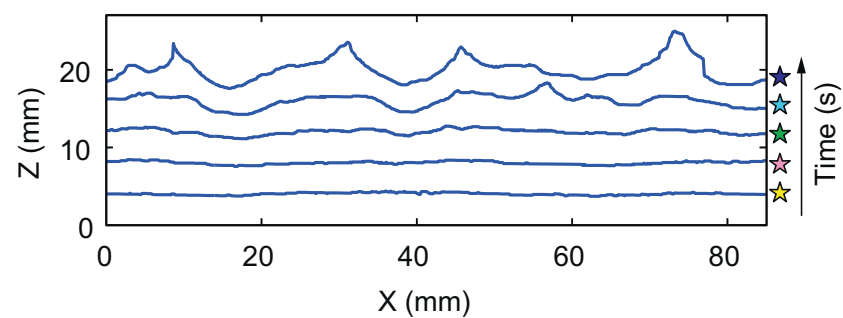

Figure 3 An experiment showing the formation of a flame structure. (a) The experimental cell is shaken for $5 \mathrm{~s}$ at an acceleration of $40.5 \mathrm{~m} / \mathrm{s}^{2}$, a frequency of $40 \mathrm{~Hz}$, and an amplitude of $0.64 \mathrm{~mm}$. The two yellow lines trace the surface of the granular layer and the two-layer interface. The two light blue reference lines at $5 \mathrm{~s}$ indicate these heights at $t=0 \mathrm{~s}$. (b) A close-up of the two-layer interface at $t=1.6 \mathrm{~s}$ indicated by a rectangle in (a). The measured wavelength of the instability is $\lambda=3 \pm 1 \mathrm{~mm}$ (average and standard deviation for three wavelengths). (c) Same as (b) but at $t=5 \mathrm{~s}$ with $\lambda=21 \pm 6 \mathrm{~mm}$ (average of three wavelengths). (d) Time evolution of the two-layer interface topography with time. The stars indicate the same timing as shown in (a). Note that the amplitude and the wavelength increase with time.

reference and subtract it from each height data. We then subtract the linear fit to the data and define the amplitude by its standard deviation. The figure shows that the initial amplitude is $\delta z \simeq 0.068 \mathrm{~mm}$, which is intermediate between the particle sizes which comprise the upper and lower layers. The amplitude initially increases exponentially with time, but its growth stops at $t \sim 4.3 \mathrm{~s}$. The flame structure remains after we stop shaking at $5 \mathrm{~s}$. Here, we define the relative amplitude $\delta z^{\prime}=\delta z-\delta z_{0}$, where $\delta z_{0}$ is the amplitude at $t=0.1 \mathrm{~s}$. Using $\delta z^{\prime}$, we define the following stages I to III: stage I for $\delta z^{\prime}<0.1 \mathrm{~mm}$, stage II for $0.1 \leq \delta z^{\prime}<0.6 \mathrm{~mm}$, and stage III for $\delta z^{\prime} \geq 0.6 \mathrm{~mm}$. The threshold of $\delta z^{\prime}=0.1 \mathrm{~mm}$ is close to the spatial resolution limit of the images $(\simeq 0.12 \mathrm{~mm})$. For $\delta z^{\prime} \geq 0.6 \mathrm{~mm}$, a fully developed flame structure forms. We consider these flame structures fully developed because a peak amplitude can be identified in the amplitude vs time plot during shaking, for most (14 out of 15) of the experiments which transit to stage III. Examples of such peaks are indicated by arrows in Figures 4b, 5b, and 6.

\section{Acceleration and frequency dependence}

We next show how the resulting instability changes when the peak acceleration and frequency of shaking are varied. Figure $5 \mathrm{a}$ and Additional file 2 show the acceleration dependence at a fixed frequency of $50 \mathrm{~Hz}$. The figure shows that for a small acceleration of $2.1 \mathrm{~m} / \mathrm{s}^{2}$, the interstitial water percolates upwards, the granular layer compacts, and the instability ends in stage I. However, for the acceleration of $19.3 \mathrm{~m} / \mathrm{s}^{2}$, the instability evolves from stage I to stage II. Finally, for $40.5 \mathrm{~m} / \mathrm{s}^{2}$, the instability evolves through stages I and II, and ends in stage III. The growth of amplitudes for the three experiments shown in Figure 5a and two additional experiments at the same frequency is shown in Figure $5 \mathrm{~b}$. The figure shows that as peak acceleration increases, both the growth rate (initial 
a
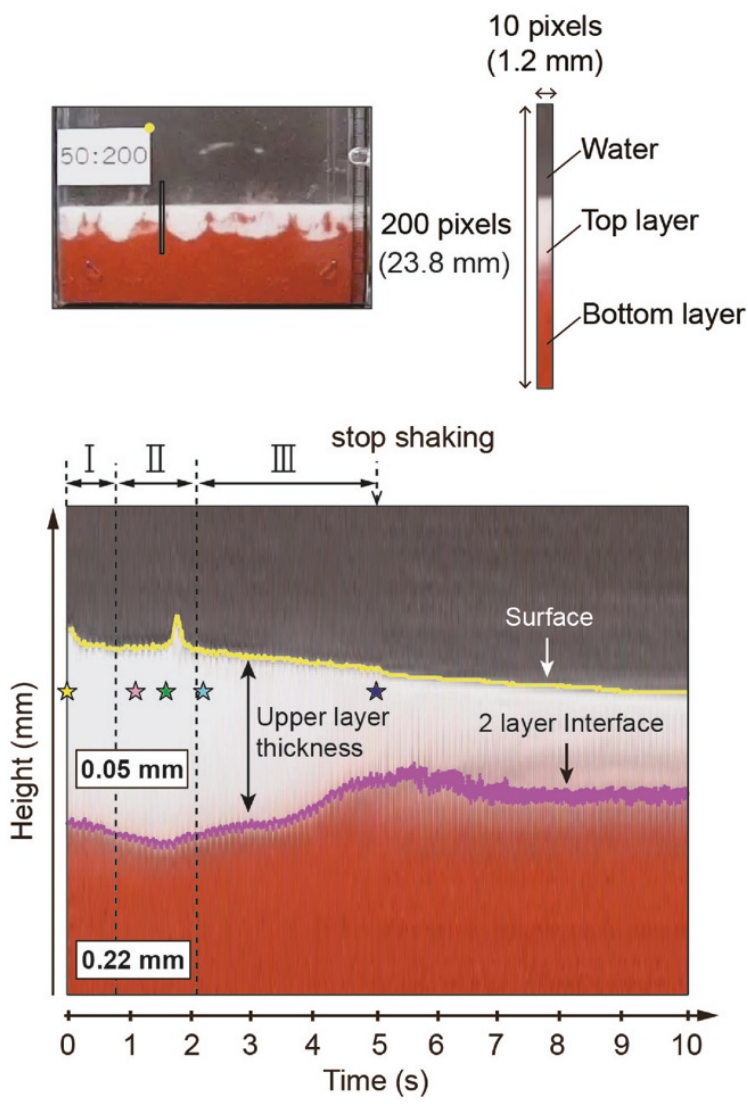

b

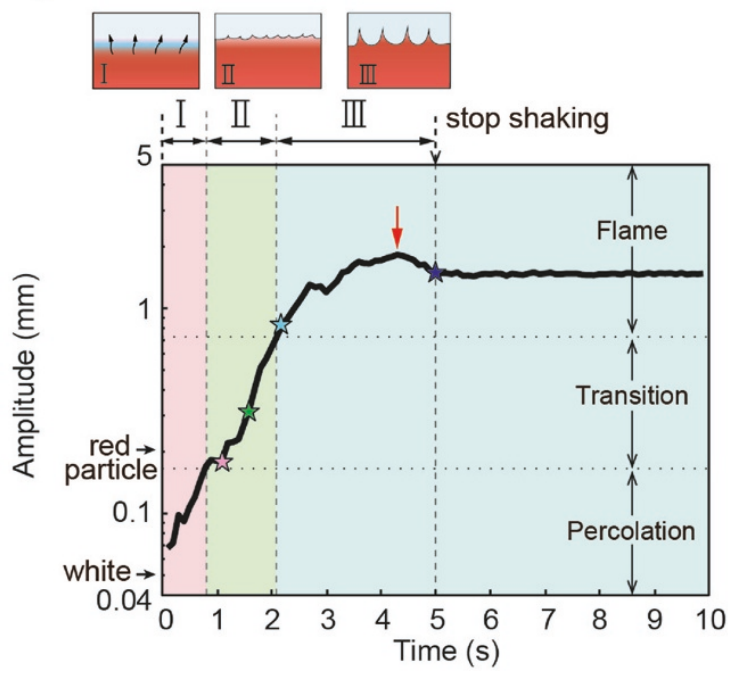

Figure 4 Time evolution of the height of the two-layer interface and the amplitude of the instability. (a) Growth of the interfacial heights with time for the same experiment shown in Figure 3. Here, a vertical section whose location is indicated in the left photo is cut from each image. The images are aligned horizontally at an interval of $0.033 \mathrm{~s}$ as a function of time. Yellow and pink lines trace the upper surface of the granular layer and the two-layer interface, respectively. I, II, and III indicate the three stages which are defined according to the relative amplitude $\delta z^{\prime}$ (see text for the details). Stars correspond to the same timing as shown in Figure 3a. (b) Growth of the amplitude $(\delta z)$ with time for the same experiment shown in (a). The three stages are defined according to the relative amplitude $\left(\delta z^{\prime}\right)$ values of 0.1 and $0.6 \mathrm{~mm}$. Diameters of the red and white particles which comprise the lower and upper layers, respectively, are indicated for comparison. The amplitude initially increases exponentially with time. At $t \sim 4.3 \mathrm{~s}$, the upper tip of the flame structure detaches and the amplitude slightly decreases. A red arrow indicates the peak amplitude. This amplitude remains after the shaking stops at $5 \mathrm{~s}$.

slope of the curves in Figure 5b) and the amplitude of the instability increase.

Figure 6 shows the frequency dependence at a fixed acceleration of $5.1 \pm 0.3 \mathrm{~m} / \mathrm{s}^{2}$, where the error is given by the standard deviation. Additional file 3 shows four selected examples from the experiments shown in Figure 6 . Here, we note that at a constant acceleration, the amplitude $(A)$ of shaking decreases with frequency $(f)$ as $A \propto f^{-2}$. Figure 6 shows that at a frequency of $150 \mathrm{~Hz}$, the instability growth is fastest and attains the largest amplitude.

We conducted a total of 73 experiments with different combinations of shaking acceleration and frequency, and classified the results into the following four regimes using the relative amplitude $\delta z^{\prime}$ and the total compaction of the whole granular layer $\delta h$ at $t=9.9 \mathrm{~s}$ (i.e., $4.9 \mathrm{~s}$ after the shaking stops): regime Ia (no change), when $\delta z^{\prime}<0.1 \mathrm{~mm}$ and $\delta h<0.1 \mathrm{~mm}$; regime $\mathrm{Ib}$ (percolation), when $\delta z^{\prime}<$ $0.1 \mathrm{~mm}$ and $\delta h \geq 0.1 \mathrm{~mm}$; regime II (transition), when $0.1 \leq \delta z^{\prime}<0.6 \mathrm{~mm}$; and regime III (flame), when $\delta z^{\prime} \geq$ $0.6 \mathrm{~mm}$. Here, regimes I to III are defined using the same threshold values of $\delta z^{\prime}$ used to define stages I to III, but at $t=9.9 \mathrm{~s} . \delta h$ is used to subdivide regime I into regimes Ia and Ib. For all experiments in regimes II to III, $\delta h \geq$ $0.1 \mathrm{~mm}$, and therefore, we do not use $\delta h$ to classify these regimes. For the amplitude data shown in Figures $5 \mathrm{~b}$ and 6 , the different resulting regimes are indicated by the line thicknesses.

In Figure 7a, we plot the classified regimes using different marker shapes and $\delta z^{\prime}$ ranges using different marker sizes (colors), in the parameter space of acceleration and frequency. The figure shows that there is a critical acceleration above which the instability grows and regime Ib (percolation, in square markers) transits to regime II (transition, in triangle markers). In addition, we find 


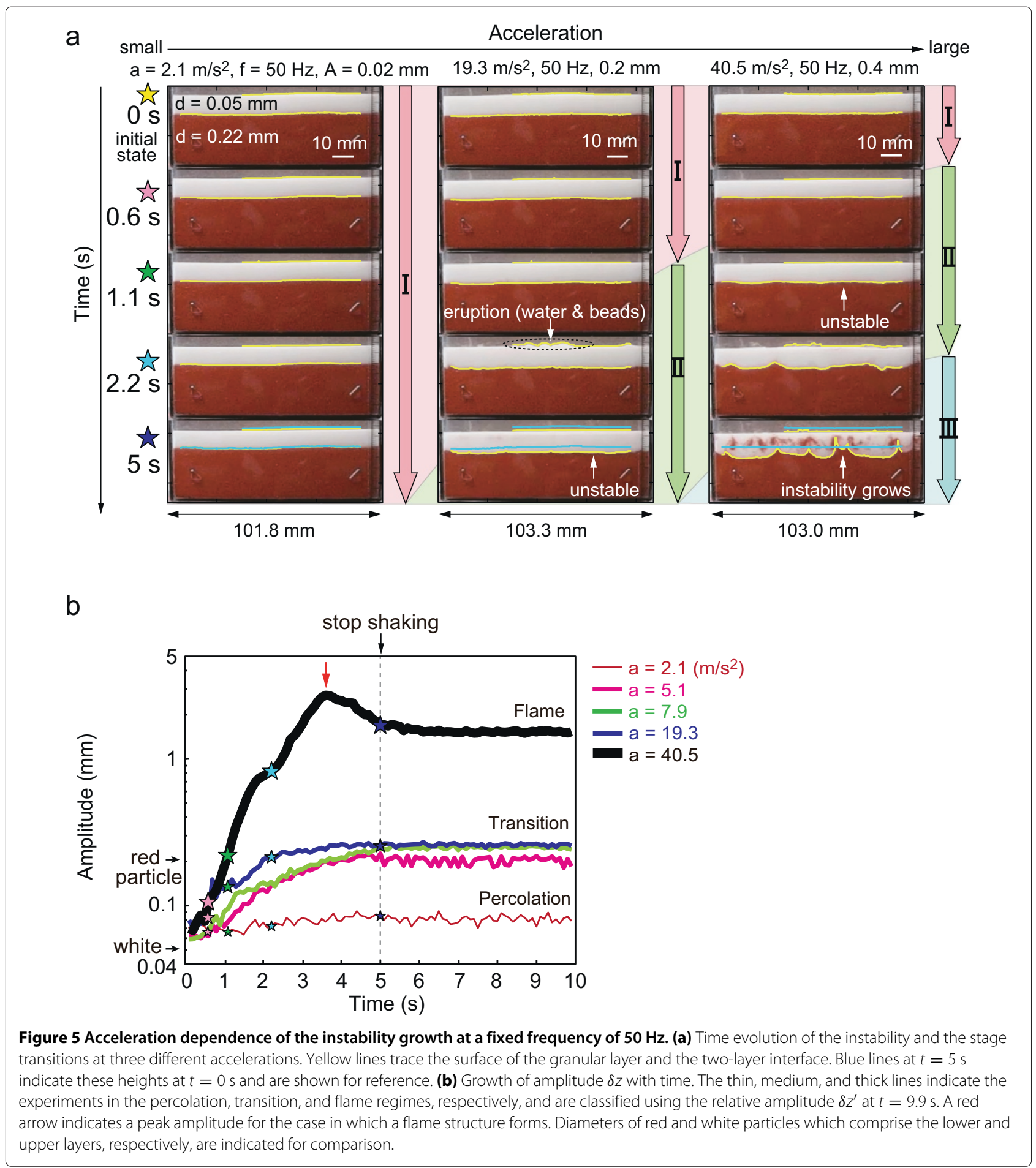

that the critical acceleration is frequency dependent and is minimum $\left(3.4 \mathrm{~m} / \mathrm{s}^{2}\right)$ at a frequency of approximately $100 \mathrm{~Hz}$.

In Figure $7 \mathrm{~b}$, we show the same regime diagram using the dimensionless parameters. Here, we nondimensionalize acceleration by the reduced gravity $(\Delta \rho g / \rho)$ to obtain a dimensionless acceleration

$$
\Gamma=\frac{\ddot{z}_{\text {peak }}}{\Delta \rho g / \rho}=\frac{A(2 \pi f)^{2}}{\Delta \rho g / \rho},
$$

where $\ddot{z}_{\text {peak }}=A(2 \pi f)^{2}$ is the peak acceleration. Similarly, we non-dimensionalize frequency by $V_{\mathrm{s}} / d_{\mathrm{s}(\mathrm{sm})}$ to obtain a dimensionless frequency

$$
f^{\prime}=\frac{d}{V_{\mathrm{s}(\mathrm{sm})}} f .
$$




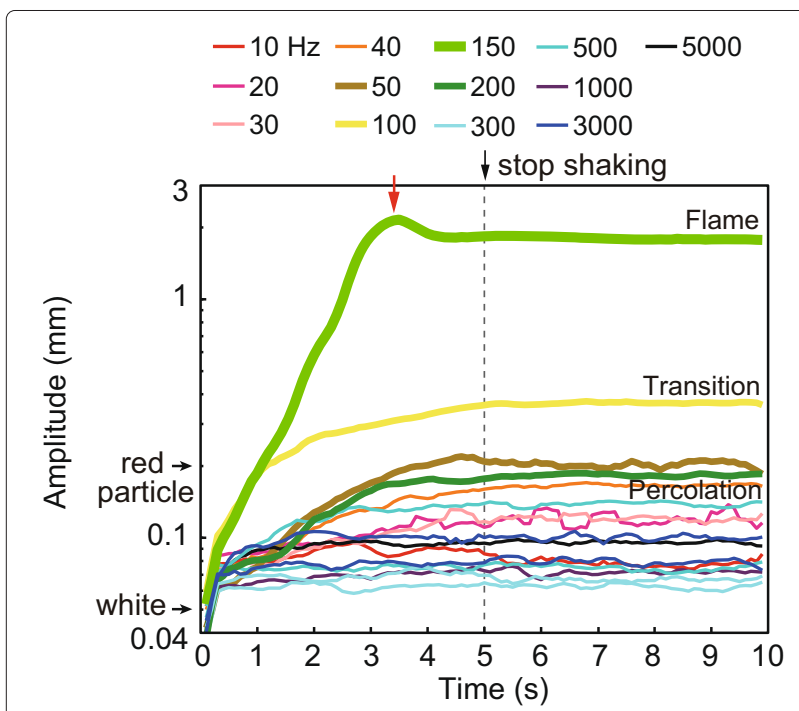

Figure 6 Frequency $(f)$ dependence of amplitude $(\delta z)$ growth in the range of $f=10-5,000 \mathrm{~Hz}$ at fixed acceleration of $5.1 \pm 0.3$ $\mathrm{m} / \mathrm{s}^{2}$. The thin, medium, and thick lines correspond to the percolation, transition, and flame regimes, which are classified using the relative amplitude $\delta z^{\prime}$ at $t=9.9 \mathrm{~s}$. The growth of the instability is fastest and attains the largest amplitude at an intermediate frequency of $150 \mathrm{~Hz}$. A red arrow indicates a peak amplitude for the case in which a flame structure forms. Diameters of red and white particles which comprise the lower and upper layers, respectively, are indicated for comparison. Here, the amplitude data are smoothed (five-point running average) for the ease of visualization.

Similar non-dimensionalizations have been used previously (e.g., Pak and Behringer 1993; Melo et al. 1995; Duran 2000; Schleier-Smith and Stone 2001; Burtally et al. 2002; Voth et al. 2002; Leaper et al. 2005; Eshuis et al. 2005, 2007). Here, we define the onset of instability when the relative amplitude is $\delta z^{\prime} \geq 0.1 \mathrm{~mm}$ (i.e., onset of the transitional regime). Figure $7 \mathrm{~b}$ indicates that the minimum acceleration for the instability to occur is $\Gamma=0.58$ at a frequency of $f^{\prime}=2.5$.

In Figure 8, we plot the relative amplitudes $\delta z^{\prime}$ at $t=$ $9.9 \mathrm{~s}$, which were used to classify the regimes, as a function of acceleration $\ddot{z}_{\text {peak }}$ for five selected frequencies in the range of 40 to $200 \mathrm{~Hz}$. The figure shows that the transition from percolation to flame regime is continuous. In particular, for the $100 \mathrm{~Hz}$ data, we obtain $\delta z^{\prime} \propto\left(\ddot{z}_{\text {peak }}\right)^{1.04}$, with an exponent close to a linear dependence.

\section{Effects of other parameters}

In addition to the shaking conditions, there are many other changeable parameters in our experiments. Here, we briefly describe the effects of other parameters which help to better understand the dynamics occurring in our experiments. Here, we define the experiments for the parameters shown in Figure 2 as the reference case.

First, we describe the results of two dry experiments (i.e., without water) and otherwise the same as the reference

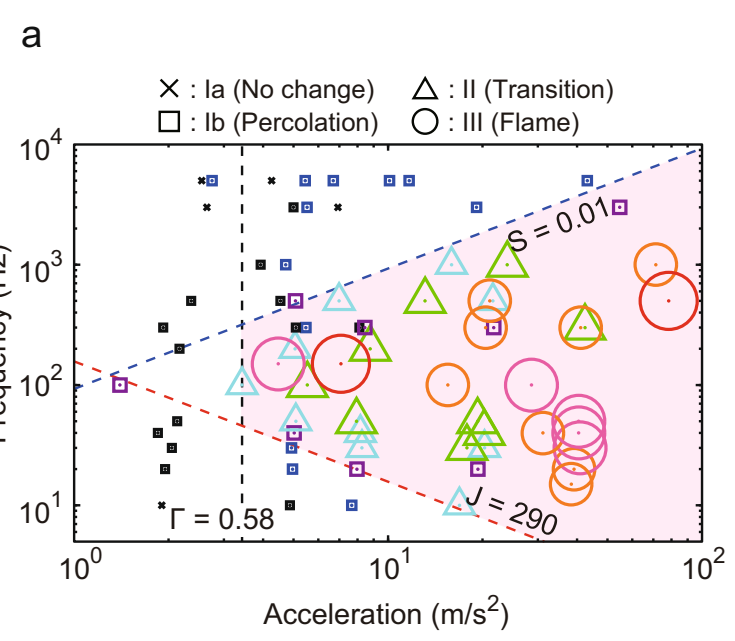

b

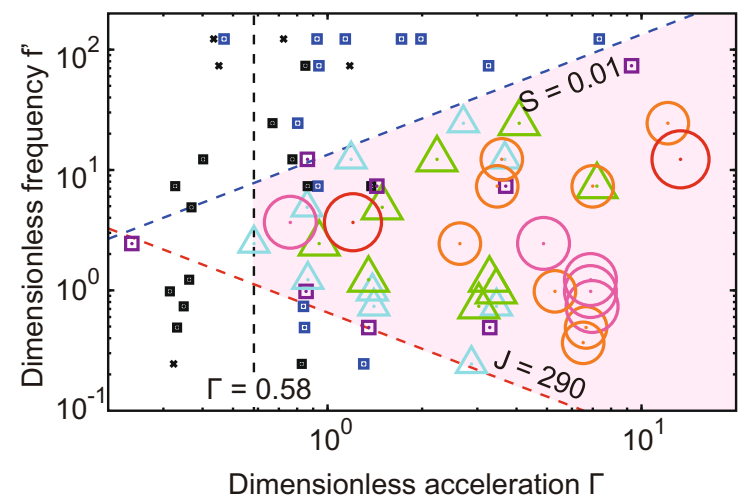

Figure 7 Regime diagram of the experiments. (a) Plotted in the parameter space of acceleration and frequency. The four regimes (in different marker shapes) are as follows: la, no change (crosses); Ib, percolation (squares); II, transition (triangles); and III, flame (circles). These are classified according to the relative amplitude $\delta z^{\prime}$ (see text for details). The marker sizes (and colors) correspond to the magnitude of $\delta z^{\prime}$ (in $\mathrm{mm}$ ) at $t=9.9 \mathrm{~s}$ and are classified as follows. Squares and crosses in black $\delta z^{\prime}<0.01$. Squares in blue $0.01 \leq \delta z^{\prime}<0.05$ and in purple $0.05 \leq \delta z^{\prime}<0.1$. Triangles in light blue $0.1 \leq \delta z^{\prime}<0.15$ and in green $0.15 \leq \delta z^{\prime}<0.6$. Circles in orange $0.6 \leq \delta z^{\prime}<1.3$, in pink $1.3 \leq \delta z^{\prime}<2.0$, and in red $\delta z^{\prime} \geq 2.0$. The black, blue, and red broken lines indicate the critical acceleration of $3.4 \mathrm{~m} / \mathrm{s}^{2}$ (corresponds to a dimensionless acceleration $\Gamma_{\mathrm{c}}=0.58$, where $\Gamma$ is defined by Equation 3), the critical shaking strength $S_{C}=0.01$ (corresponds to Equation 6 where $S$ is defined in Equation 5), and the critical jerk strength $J_{C}=290$ (corresponds to Equation 8 with $J$ defined in Equation 7), respectively. The red colored domain indicates the conditions under which these three dimensionless parameters are supercritical (i.e., $\Gamma>\Gamma_{c}, S>S_{C}$, $J>J_{c}$ ). (b) Same as (a) but using dimensionless parameters $\Gamma$ and $f^{\prime}$ (Equation 4).

case. We find that the results are strikingly different. Most importantly, flame structure does not form in the dry experiments. At $\ddot{z}_{\text {peak }}=20.7 \mathrm{~m} / \mathrm{s}^{2}$ and $f=50 \mathrm{~Hz}$, the small particles comprising the upper layer settle into the 


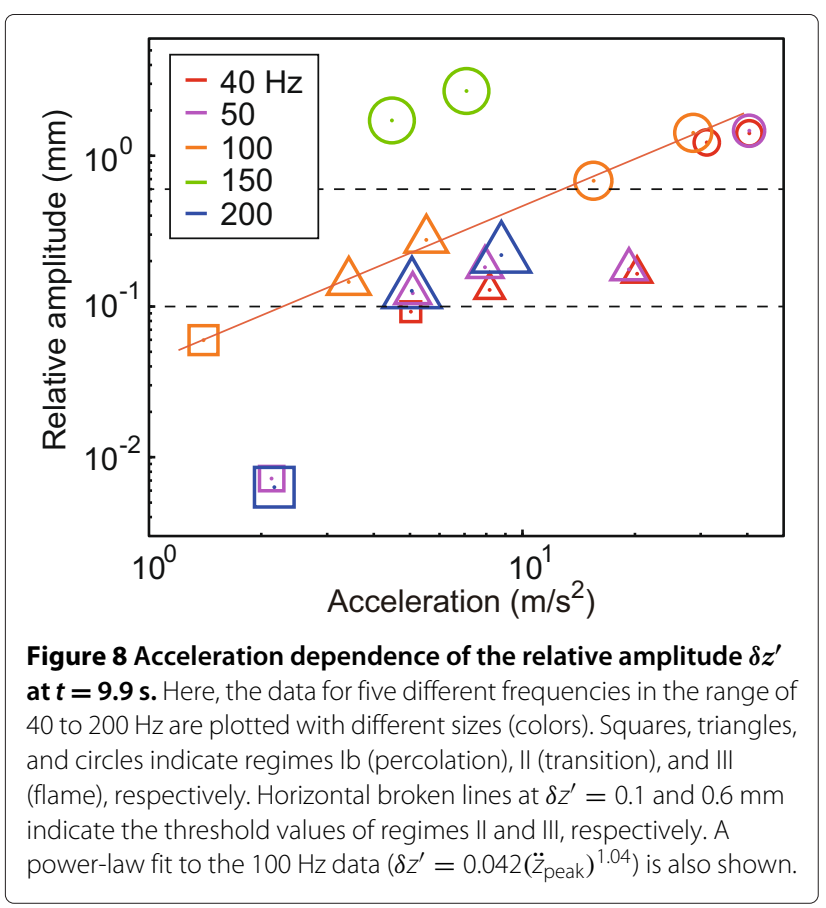

pore space of the large particles comprising the lower layer and formed a mixture layer whose thickness increased with time. Furthermore, the granular layer as a whole gradually tilts. At $\ddot{z}_{\text {peak }}=39.6 \mathrm{~m} / \mathrm{s}^{2}$ and $f=50 \mathrm{~Hz}$, in addition to tilting, a large-scale whole layer granular convection with a length scale comparable to the case width occurs.

Second, we describe the results of experiments using a wider case (width $194 \mathrm{~mm}$, height $104 \mathrm{~mm}$, thickness $26 \mathrm{~mm}$ ) and otherwise the same as the reference case. From a series of experiments at fixed accelerations of $\ddot{z}_{\text {peak }}=5.0$ and $8.8 \mathrm{~m} / \mathrm{s}^{2}$ and different frequencies, we find that the final amplitude reached a maximum at a frequency of approximately $100 \mathrm{~Hz}$, the same as the reference case. We also find a similar frequency dependence of the critical acceleration and a similar wavelength of the instability of $\lambda \sim 10 \mathrm{~mm}$, indicating that the effects of width dimension are not evident.

Third, we describe the results of different layer thicknesses. When there is no permeability barrier (i.e., one layer consisting of a particle size $d=0.22 \mathrm{~mm}$ with a thickness $21.9 \mathrm{~mm}$ ), neither sand boil nor flame structure formation occurs. We also compare three experiments with the same thickness $33.8 \pm 2.0 \mathrm{~mm}$ of the granular layer but with different thickness fractions of the upper layer: $0.19,0.28$, and 0.78 . For $\ddot{z}_{\text {peak }}=40.0 \pm 0.8 \mathrm{~m} / \mathrm{s}^{2}$ and $f=50 \mathrm{~Hz}$, for all three cases, a similar flame structure forms. However, we find that the relative amplitude at $t=9.9 \mathrm{~s}$ decreases by $35 \%$ as the upper layer becomes thicker.

\section{Discussion}

\section{The origin of the flame structure}

We consider that the flame structure in our experiments forms as a consequence of Rayleigh-Taylor type instability and not from surface instability, resonance, or granular convection. There seem to be at least three requirements for Rayleigh-Taylor instability to occur: the presence of a permeability barrier, a large fluid viscosity, and granular matter being dense. We explain each of these in detail below.

First, whenever a flame structure developed, it always formed at the permeability barrier. This indicates that the accumulation of water at the permeability barrier is necessary for the flame structure to form, from which we infer that the instability is of Rayleigh-Taylor type.

Second, granular convection did not occur in the waterimmersed experiments, whereas it did occur in the dry experiments. On the other hand, in the dry experiments, the small particles comprising the upper layer settled downwards and the flame structure did not form. Here, we evaluate the importance of viscosity by comparing the terminal velocity given by the Stokes velocity $V_{\mathrm{s}}$ (Equation 1 ) and the free fall velocity $V_{\mathrm{f}}=\sqrt{2(\Delta \rho / \rho) g d}$ where the particle diameter scale $d$ is taken as the height scale. The ratio of these two velocity scales is the Stokes number $S t=V_{\mathrm{s}} / V_{\mathrm{f}}$ (e.g., Andreotti et al. 2013). If $S t \ll 1$, viscous drag is large such that the terminal velocity is attained within a particle diameter scale (viscous regime), and vice versa for $S t \gg 1$ (free-fall regime). If we use the particle size $d$ of the upper layer $(d=0.05 \mathrm{~mm})$, for the waterimmersed case, we obtain $S t \sim 0.08<1$. In contrast for the dry case, we obtain $S t \sim 6>1$. This suggests that the suppression of particle motions by viscosity played a role for the accumulation of water at the permeability barrier.

Third, in our experiments, the particles were initially closely packed, and even when they were shaken, we did not observe any standing surface waves indicating that the particles remained close to a close packed state. Even for the dry experiments, granular matter did not detach from the bottom of the case or bounce (e.g., Eshuis et al. 2007). The thickness of the whole layer scaled by the particle size is $N \sim 270$. A large value of $N$ results in a larger frictional dissipation (Eshuis et al. 2007), which causes the granular matter to remain dense even when shaken.

\section{An interpretation of a regime diagram}

Here, we consider the origin of the critical acceleration and its frequency dependence. Our experiments indicate that the instability occurs when $\Gamma>\Gamma_{\mathrm{c}}=0.58$, which we indicate by vertical lines in Figure $7 \mathrm{a}, \mathrm{b}$. The critical value $\Gamma_{\mathrm{c}}=0.58$ can be understood in terms of the force balance. The inertial stress of a granular layer is $\sim \rho h \ddot{z}_{\text {peak }}=\rho A h(2 \pi f)^{2}$ where $h$ is the thickness scale. 
Frictional stress is $\sim \mu \Delta \rho g h$, where $\mu$ is the friction coefficient. $\mu$ estimated from the angle repose $\theta_{\mathrm{r}}$ of a pile of glass beads is $\mu=\tan \theta_{\mathrm{r}} \sim 0.4$ to 0.5 (Samadani and Kudrolli 2001; Higashi and Sumita 2009). It follows that the ratio of the inertial stress to the frictional stress is $\sim \Gamma / \mu$, where we used the definition for $\Gamma$ (Equation 3). Inertial stress must exceed the frictional stress to displace a particle from which we expect $\Gamma>\mu$ is required for instability to occur. Our experimental result of $\Gamma>0.58$ for instability to occur is consistent with $\Gamma>\mu \sim 0.4$ to 0.5 .

Next, we consider the frequency dependence. Here, we show that the high frequency limit can be understood in terms of energy balance, which is the integrated form of the force balance equation. For liquefaction to occur, the energy of shaking must be sufficiently large so that a particle can be lifted against gravity. If we use the particle diameter $d$ for the height scale, the ratio of the energy of shaking to the work needed to lift a particle against gravity is expressed by the dimensionless shaking strength $S$ (Pak and Behringer 1993; Eshuis et al. 2005, 2007):

$$
S=\frac{A \ddot{z}_{\text {peak }}}{\Delta \rho g d / \rho}=\frac{A^{2}(2 \pi f)^{2}}{\Delta \rho g d / \rho}=\left(\frac{1}{\Delta \rho g d / \rho}\right)\left(\frac{\ddot{z}_{\text {peak }}}{2 \pi f}\right)^{2}
$$

A criterion can then be defined as $S>S_{\mathrm{c}}$ where $S_{\mathrm{c}}$ is the critical value, which can be rewritten using Equation 5 as

$$
f<\left(\frac{\rho}{\Delta \rho g d S_{\mathrm{c}}}\right)^{1 / 2} \cdot\left(\frac{\ddot{z}_{\text {peak }}}{2 \pi}\right) .
$$

We use Equation 6 and draw a critical line in Figure 7a,b for $S_{\mathrm{c}}=0.01$, which separates regimes Ib and II fairly well. We note however that the high frequency limit may alternatively be interpreted in terms of critical shaking velocity $v_{\mathrm{c}}$ (Hejmandy et al. 2012), since the critical velocity line also scales as $f \propto \ddot{z}_{\text {peak }} / v_{\mathrm{c}}$.

The low frequency limit can be understood in terms of jerk, which is a time derivative of acceleration. In the limit of very low frequency, the acceleration can be approximated as constant. We propose that the change of acceleration (i.e., $\left.d^{3} z / d t^{3} \propto A(2 \pi f)^{3}\right)$ is important for liquefaction because it causes relative motion between the particles. On the other hand, in the absence of shaking, the characteristic scale for the change of acceleration of a particle settling in a viscous fluid is $\sim V_{\mathrm{s}}^{3} / d^{2}$. We use this scale to non-dimensionalize jerk and define the jerk strength $J$

$$
J=\left(\frac{d^{3} z}{d t^{3}}\right)_{\text {peak }}\left(\frac{d^{2}}{V_{\mathrm{s}}^{3}}\right)=\frac{A(2 \pi f)^{3}}{V_{\mathrm{s}}^{3} / d^{2}}=\left(\frac{2 \pi f}{V_{\mathrm{s}}^{3} / d^{2}}\right) \ddot{z}_{\text {peak }} .
$$

A criterion $J>J_{\mathrm{c}}$ can then be rewritten as

$$
f>\left(\frac{J_{\mathrm{c}}}{2 \pi} \frac{V_{\mathrm{s}}^{3}}{d^{2}}\right)\left(\ddot{z}_{\text {peak }}\right)^{-1} \text {. }
$$

In Figure 7a,b, we draw a line for $J_{\mathrm{c}}=290$, which separates regimes Ib and II fairly well. The conditions under which these three dimensionless parameters are supercritical, i.e., $\Gamma>\Gamma_{\mathrm{c}}, S>S_{\mathrm{c}}$, and $J>J_{\mathrm{c}}$, correspond to the colored domain bounded by the three lines in Figure 7a,b.

Finally, we derive the frequency of approximately $100 \mathrm{~Hz}$ at which the critical acceleration is minimum. From combining the conditions $S_{\mathrm{c}} \sim 0.01, J_{\mathrm{c}} \sim 290$, and $\Gamma \sim 0.22$, which is the value of $\Gamma$ at the intersection of the critical $S, J$ lines (see Figure 7b), we obtain $f^{\prime}=f \cdot d / V_{\mathrm{s}} \sim 3$, which corresponds to approximately $100 \mathrm{~Hz}$. We also obtain $A \sim 0.05 d$ which is the amplitude of shaking relative to the particle size comprising the upper layer under this condition.

\section{Comparison with the sedimentary rocks}

Our experimental parameters (particle size pair, densities, layer thickness ratios) were not chosen to closely match or scale those of the sedimentary rocks in Figure 1. Therefore, our regime diagram (Figure 7) cannot be directly applied to these outcrops. If one wishes to constrain the exact shaking condition required for the flame structure in these outcrops to form, a similar set of experiments using the same particles from these outcrops need to be conducted. Nevertheless, we consider that the basic features of the regime diagram will remain unchanged.

Here, we point out several features which are common with the flame structures in Figure 1 and in Figure 3. First is that they both have upward pointing cusps. Similar cusps have been previously observed in RayleighTaylor instability of a liquid-immersed granular medium (e.g., Michioka and Sumita 2005; Shibano et al. 2012). In addition, for both cases, the preserved flame structures do not fully penetrate through the upper layer. Second is that in the experiments, even when liquefaction occurs, only the uppermost part of the lower layer seems to be fluidized. We may approximate the situation in which water accumulates beneath the permeability barrier as a thin low viscosity layer with a thickness $\delta$ underlying a thick high viscosity layer. Here, we use the results of linear stability analysis (Whitehead and Luther 1975), from which we can constrain $\delta$ from the wavelength of instability $\lambda$ as $\delta<\lambda / \pi$. It follows that for $\lambda \sim 21 \mathrm{~mm}, \delta<7 \mathrm{~mm}$, implying that only the uppermost part of the coarse lower layer is fluidized. Similarly, for the outcrops, using the wavelength of $\lambda \sim 40 \mathrm{~mm}$, we obtain $\delta<13 \mathrm{~mm}$, suggesting that only the uppermost one fifth or less of the tuff layer (layer 2 in Figure 1) 
became fluidized. Field observations indicate that the thickness of the tuff layer varies in the range of 20 to $80 \mathrm{~mm}$, whereas the wavelength is found to be around $\lambda \sim 40 \mathrm{~mm}$. The constancy of the wavelength suggests that the thickness of the fluidized layer is also approximately constant, regardless of the thickness of the tuff layer.

\section{Conclusions}

We conducted a series of experiments in which a twolayered water-immersed granular medium, where the upper layer forms a permeability barrier, is shaken vertically at different combinations of accelerations and frequencies. We find that above a critical acceleration, the instability develops at the two-layer interface and grows. For a sufficiently large acceleration, a flame structure which is similar to those observed in sedimentary rocks forms. We also find that the critical acceleration is frequency dependent and is minimum at approximately $100 \mathrm{~Hz}$. In other words, there is an optimum frequency band in which the flame structure most easily develops. These results were interpreted by combined conditions of inertial stress, energy, and jerk of shaking exceeding their critical values. Although further work is needed to clarify how the optimum frequency band depends on the parameters such as fluid viscosity and particle size, our experiments suggest that the occurrence of a flame structure may be used to constrain the shaking conditions when these structures formed.

\section{Additional files}

Additional file 1: Movie 1 in QuickTime. An example of an experiment in which a flame structure forms at an acceleration of $40.5 \mathrm{~m} / \mathrm{s}^{2}$, a frequency of $40 \mathrm{~Hz}$, and an amplitude of $0.64 \mathrm{~mm}$, replayed at $\times 0.2$ speed. This is the same experiment which was shown in Figures 3 and 4.

Additional file 2: Movie $\mathbf{2}$ in QuickTime. Acceleration dependence at a fixed frequency of $50 \mathrm{~Hz}$ replayed at $\times 0.4$ speed. These are four selected examples from those shown in Figure $5 b$. Accelerations in $\mathrm{m} / \mathrm{s}^{2}$ (and the resulting regime) are 2.1 (Ib: percolation), 7.9 (II: transition), 19.3 (II: transition), and 40.5 (III: flame), respectively.

Additional file 3: Movie 3 in QuickTime. Frequency dependence at a fixed acceleration of $5.0 \pm 0.4 \mathrm{~m} / \mathrm{s}^{2}$ replayed at $\times 0.4$ speed. These are four selected examples from those shown in Figure $6 \mathrm{~b}$. Frequencies in $\mathrm{Hz}$ (and resulting regime) are 10 (Ib: percolation), 100 (II: transition), 150 (IIl: flame), and 3,000 (Ib: percolation), respectively. Note that the flame structure forms at the intermediate frequency of $150 \mathrm{~Hz}$.

\section{Competing interests}

The authors declare that they have no competing interests.

\section{Authors' contributions}

NY made the apparatus, planned and conducted the experiments, and analyzed and interpreted the results. IS contributed to setting up the apparatus and interpreting the results. Both authors contributed to the preparation of the manuscript. Both authors read and approved the final manuscript.

\section{Acknowledgements}

We thank Y. Shibano and A. Namiki for discussions and technical advice, and the two anonymous reviewers for helpful comments on the manuscript. This work was supported by KAKENHI $(22109505,24244073,24510246)$.

Received: 25 November 2013 Accepted: 28 May 2014

Published: 20 June 2014

References

Allen JRL (1985) Principles of physical sedimentology. Blackburn Press, Caldwell, New Jersey

Andreotti B, Forterre Y, Pouliquen O (2013) Granular media. Cambridge University Press, Cambridge

Burtally N, King PJ, Swift MR (2002) Spontaneous air-driven separation in vertically vibrated fine granular mixtures. Science 295:1877-1879. doi:10.1126/science.1066850

Clement CP, Pacheco-Martinez HA, Swift MR, King PJ (2009) Partition instability in water-immersed granular systems. Phys Rev E 80:011311

Clement CP, Pacheco-Martinez HA, Swift MR, King PJ (2010) The water-enhanced Brazil nut effect. EPL 91:54001. doi:10.1209/0295-5075/91/54401

Duran J (2000) Sands, powders, and grains: an introduction to the physics of granular materials. Springer, New York

Eshuis P, van der Weele K, van der Meer D, Lohse D (2005) Granular Leidenfrost effect: experiment and theory of floating particle clusters. Phys Rev Lett 95:258001

Eshuis P, van der Weele $K$, van der Meer D, Bos R, Lohse D (2007) Phase diagram of vertically shaken granular matter. Phys Fluids 19:123301

Hejmandy P, Bandyopadhyay R, Sabhapandit S, Dhar A (2012) Scaling behavior in the convection-driven Brazil nut effect. Phys Rev E 86:050301

Higashi N, Sumita I (2009) Experiments on granular rheology: effects of particle size and fluid viscosity. J Geophys Res 114:04413. doi:10.1029/2008JB005999

Kokusho T (1999) Water film in liquefied sand and its effect on lateral spread. J Geotech Geoenviron Eng 125:817-826

Kokusho T, Kojima T (2002) Mechanism for postliquefaction water film generation in layered sand. J Geotech Geoenviron Eng 128:129-137

Leaper MC, Smith AJ, Swift MR, King PJ, Webster HE, Miles NJ, Kingman SW (2005) The behaviour of water-immersed glass-bronze particulate systems under vertical vibration. Granular Matter 7:57-67. doi:10.1007/s10035-004-0185-7

Manga M, Brodsky E (2006) Seismic triggering of eruptions in the far field: volcanoes and geysers. Ann Rev Earth Planet Sci 34: 263-291. doi:10.1146/annrevearth.34.031405.125125

Manga M, Wang C (2007) Earthquake hydrology. In: Kanamori H (ed) Treatise on geophysics, vol 4, 1st edn. Elsevier, Amsterdam, pp 293-320

Mavko G, Mukerji T, Dvorkin J (1998) The rock physics handbook. Cambridge University Press, Cambridge

Melo F, Umbanhowar PB, Swinney H (1995) Hexagons, kinks, and disorder in oscillated granular layers. Phys Rev Lett 75:3838-3384

Michioka H, Sumita I (2005) Rayleigh-Taylor instability of a particle packed viscous fluid. Geophys Res Lett 32:L03309. doi:10.1029/2004GL021827

Montenat C, Barrier P, d'Estevou PO, Hibsch C (2007) Seismites: an attempt at critical analysis and classification. Sedimentary Geol 196:5-30

Moretti M, Alfaro P, Caselles O, Canas JA (1999) Modelling seismites with a digital shaking table. Tectonophysics 304:369-383

Oehler DZ, Allen CC (2010) Evidence for pervasive mud volcanism in Acidalia Planitia, Mars. Icarus 208:636-657

Pak HK, Behringer RP (1993) Surface waves in vertically vibrated granular materials. Phys Rev Lett 71:1832-1835

Pondrelli M, Rossi AP, Ori GG, van Gasselt S, Ceramicola C (2011) Mud volcanoes in the geologic record of Mars: the case of Firsoff crater. Earth Planet Sci Lett 304:511-519

Shearer PM (1999) Introduction to seismology. Cambridge University Press, Cambridge

Samadani A, Kudrolli A (2001) Angle of repose and segregation in cohesive granular matter. Phys Rev E 64:051301

Shibano Y, Namiki A, Sumita I (2012) Experiments on upward migration of a liquid-rich layer in a granular medium: implications for a crystalline magma chamber. Geochem Geophys Geosyst 13:Q03007. doi:10.1029/2011GC003994 
Schleier-Smith JM, Stone HA (2001) Convection, heaping, and cracking in vertically vibrated granular slurries. Phys Rev Lett 86:3016-3019

Vucetic M (1994) Cyclic threshold shear strains in soils. J Geotech Eng 120:2208-2228

Voth GA, Bigger B, Buckley MR, Losert W, Brenner MP, Stone HA, Gollub JP (2002) Order clusters and dynamical states of particles in a vibrated fluid. Phys Rev Lett 88:234301

Whitehead JA, Luther DS (1975) Dynamics of laboratory diapir and plume models. J Geophys Res 80:705-717

Yamaguchi A, Yoshida N, Tobita Y (2008) Estimation of thickness of liquefied layer from sand boiling pattern. J Japan Soc Civil Eng Div C 64:79-89

doi:10.1186/2197-4284-1-13

Cite this article as: Yasuda and Sumita: Shaking conditions required for

flame structure formation in a water-immersed granular medium. Progress in Earth and Planetary Science 2014 1:13.

\section{Submit your manuscript to a SpringerOpen ${ }^{\circ}$ journal and benefit from:}

- Convenient online submission

- Rigorous peer review

- Immediate publication on acceptance

- Open access: articles freely available online

- High visibility within the field

- Retaining the copyright to your article

Submit your next manuscript at $\gg$ springeropen.com 